\title{
Stellar acoustic radii and ages from seismic inversion techniques
}

\author{
G. Buldgen ${ }^{1}$, D. R. Reese ${ }^{2}$, M. A. Dupret ${ }^{1}$, and R. Samadi ${ }^{3}$ \\ 1 Institut d'Astrophysique et Géophysique, University of Liège, Liège, Belgium \\ 2 School of Physics and Astronomy, University of Birmingham, Birmingham, United Kingdom \\ 3 LESIA, Observatoire de Paris, Université Denis Diderot, Meudon, France
}

\begin{abstract}
Determining stellar characteristics such as the radius, mass or age is crucial and asteroseismology is currently the most promising tool to provide these results accurately. We extend the SOLA inversion technique to new global characteristics in addition to the mean density [2] and apply our methodology to the acoustic radius and an age indicator based on the sound speed derivative. The results from SOLA inversions are compared with estimates based on the small and large frequency separations for several test cases. We show that SOLA inversions yield more accurate results than other techniques which are more sensitive to surface effects.
\end{abstract}

\section{Theoretical approach}

The inversion procedure relies on the variational principle and the frequency-structure relation for adiabatic stellar oscillations. The relative perturbation of a global characteristic $A$ (e.g. $\bar{\rho}, \tau, \ldots)$ can be related to structural variables as follows:

$$
\frac{\delta A}{A}=\int_{0}^{1} \mathcal{T}(x) \frac{\delta s_{1}}{s_{1}} d x+\int_{0}^{1} \mathcal{T}_{\text {cross }}(x) \frac{\delta s_{2}}{s_{2}} d x
$$

$\mathcal{T}$ and $\mathcal{T}_{\text {cross }}$ being the target functions. In the SOLA method, we minimise a cost function related to the target functions and the observational errors to find the optimal frequency combination to reproduce $\frac{\delta A_{o b s}}{A}$. We apply inversion techniques to the acoustic radius, $\tau$, and the age indicator, $t$, which are defined and related to the large and small frequency separations as follows:

$$
\tau=\int_{0}^{R} \frac{d r}{c} \propto \frac{1}{2 \Delta v} \quad t=\int_{0}^{R} \frac{1}{r} \frac{d c}{d r} d r \propto \frac{-4 \pi^{2} v \tilde{\delta} v}{(4 \ell+6) \Delta v}
$$

where $\ell$ is the degree of the mode, $\Delta v$ the large frequency separation and $\tilde{\delta} v$ the small frequency separation. SOLA inversions are compared with other techniques based on these asteroseismic indices. The quality of the inversion depends on how well the averaging and cross-term kernels fit their respective targets.

\section{Results for a grid of models}

We use 93 main sequence and pre-main sequence models [1] to determine the values of the $\tau$ and $t$ indicators in target $A^{\prime}$ from Reese et al. $2012\left(\mathrm{M}=0.9 \mathrm{M}_{\odot}\right.$, age $=1.492 \mathrm{Gyr}$, We used 33 frequencies with $\ell=0-2$ and $n=15-25$. The results are presented in figure 1 . The vertical lines give the position of the best model from the grid in terms of $\tilde{\delta} v$ and $\Delta v$. Each point gives the result for a given reference model of the grid and the horizontal green line corresponds to the target value. SOLA inversions yield better results when surface effects are present. Estimates based on seismic indices sometimes benefit from fortuitous error compensation, thereby leading to accurate results.

This is an Open Access article distributed under the terms of the Creative Commons Attribution License 4.0, which permits unrestricted use, distribution, and reproduction in any medium, provided the original work is properly cited. 

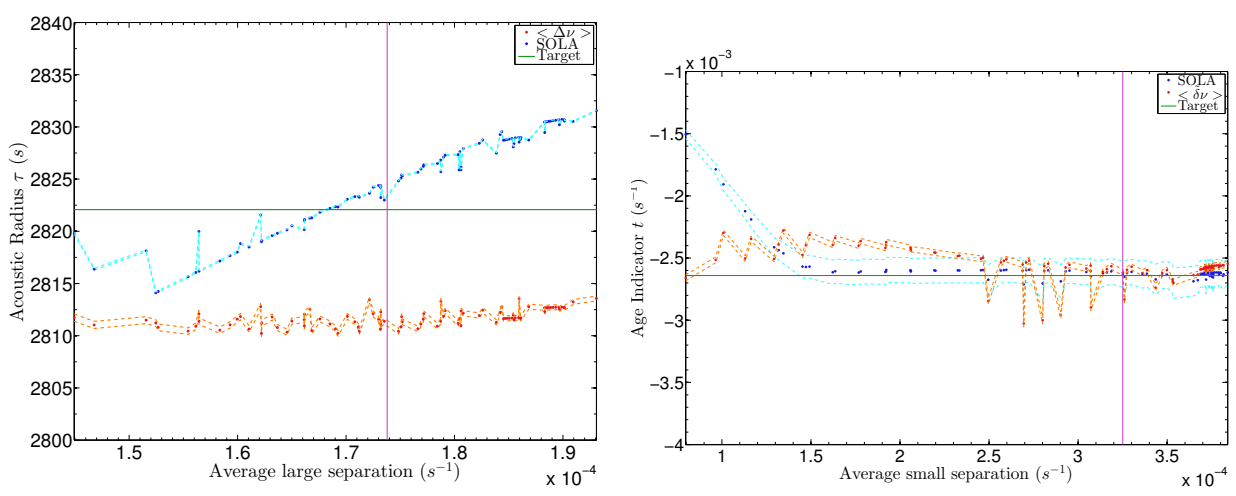

Figure 1. Results for the acoustic radius (left) and the age indicator (right) for $A^{\prime}$.

\section{Results using forward modelling}

The results for $t$ show that we need a criterion for selecting a reference model. Indeed, the inversion equations rely on the variational principle, which assumes that the reference model is close to the star. Therefore, the reference model has to be chosen carefully and we propose to obtain this model through forward modelling. Using the OSM software, we fit $\langle\Delta v\rangle$ and $\tilde{\delta} v(v)$ for the same modes as in the previous tests. 3 targets are used, with masses ranging from $0.95 \mathrm{M}_{\odot}$ to $1.05 \mathrm{M}_{\odot}$ and ages ranging from 1.5 Gyr to 6 Gyr. They differ from the reference models by their mixing-length parameter $\left(\operatorname{Model}_{\alpha_{\text {conv }}}\right)$, the presence of turbulent pressure (Model $\mathrm{turb}_{\text {rb }}$ ) or non-adiabatic effects in their frequencies $\left(\operatorname{Model}_{\text {nad }_{1,2}}\right)$. Results for $\tau$ and $t$ inversions are plotted in the figure 2. The target is in green, the reference model obtained with forward modelling is in black, the SOLA result is in blue, the result using asteroseismic indices is in red.
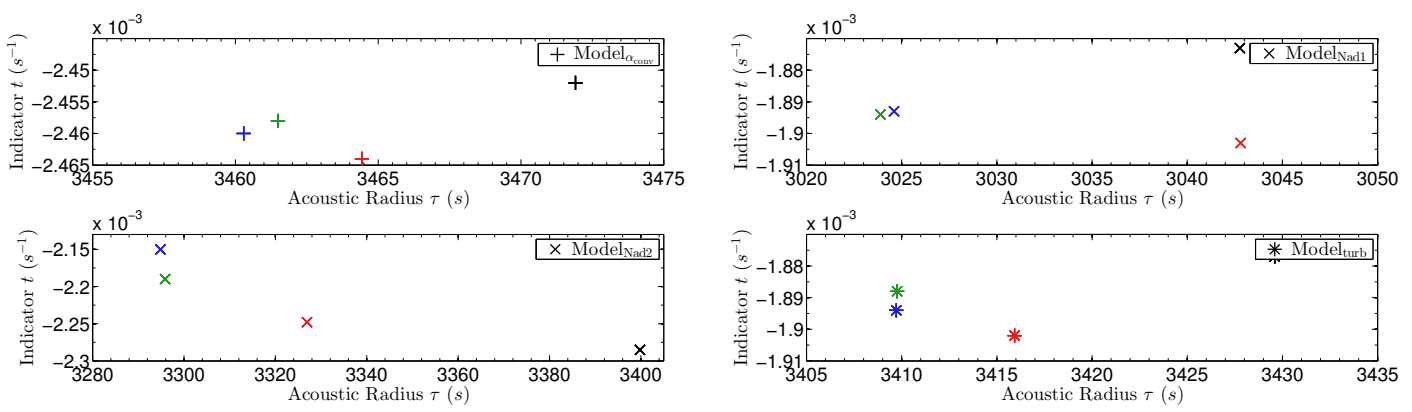

Figure 2. Results using forwar modelling.

\section{Conclusion}

The combination forward modelling + SOLA inversions can be used to accurately determine the indicators $t, \tau$ and $\bar{\rho}$ (not presented here) in observed stars. SOLA inversions always improve the accuracy with which the indicators are determined. The SOLA approach is able to overcome limitations of forward modelling, is more accurate than estimates based on asteroseismic indices and allows us to choose the structural characteristics we wish to determine.

\section{References}

1. Marques et al., Ap\&SS 316, (2008) 173-178

2. Reese et al., A\&A 316, (2012) A63 